\title{
Recent Advancements in Hematology: Knowledge, Methods and Dissemination
}

Jude Fitzgibbon ${ }^{1}$, Alessandro Busca ${ }^{2}$, Annunziata Gloghini ${ }^{3}{ }^{(D}$, Armando López-Guillermo ${ }^{4}$, Attilio Orazi ${ }^{5}$, Carmelo Carlo-Stella ${ }^{6}$ D , Emmanuel Andrès ${ }^{7}$ (D), Eric Van Breda ${ }^{8}$, Fabiana Busti ${ }^{9}$ (D) , Laurent Garderet ${ }^{10}$, Maurizio Martini ${ }^{11}{ }^{\mathbb{D}}$, Riccardo Dolcetti ${ }^{12}$ (D), Salvatore De Rosa ${ }^{13}\left(\mathbb{D}\right.$, Sergio Siragusa ${ }^{14}$, Teresa Marafioti ${ }^{15}(\mathbb{D})$ and Antonino Carbone ${ }^{16, *,+(\mathbb{D})}$

1 Centre for Haemato-Oncology, Barts Cancer Institute, QMUL, London EC1M 6BE, UK; j.fitzgibbon@qmul.ac.uk

2 Azienda Ospedaliera-Universitaria Città della Salute e della Scienza di Torino, 10133 Turin, Italy; abusca@cittadellasalute.to.it

3 Department of Diagnostic Pathology and Laboratory Medicine, Fondazione IRCCS, Istituto Nazionale dei Tumori di Milano, Via G. Venezian 1, I-20133 Milano, Italy; annunziata.gloghini@istitutotumori.mi.it

4 Department of Hematology, Hospital Clinic de Barcelona, Villarroel 170, 08036 Barcelona, Spain; ALOPEZG@clinic.cat

5 Department of Pathology, P.L. Foster School of Medicine, Texas Tech University Health Sciences Center, El Paso, TX 79409, USA; Attilio.Orazi@ttuhsc.edu

6 Department of Biomedical Sciences, Humanitas University, Section Chief Lymphoid Malignancies, Humanitas Clinical \& Research Center, Via A. Manzoni 56, 20089 Rozzano (Milano), Italy; carmelo.carlostella@hunimed.eu

7 Department of Internal Medicine, Diabetes and Metabolic Diseases, University Hospital of Strasbourg, 1 Porte de l’Hôpital, 67091 Strasbourg Cedex, France; emmanuel.andres@chru-strasbourg.fr

8 Department of Rehabilitation Sciences \& Physiotherapy, Antwerp Multidisciplinary Metabolic Research Unit (AM2RUN), University of Antwerp, B-2000 Antwerp, Belgium; eric.vanbreda@uantwerpen.be

9 Department of Medicine, Section of Internal Medicine, Azienda Ospedaliera Universitaria Integrata Verona, University of Verona, 37129 Verona, Italy; fabiana.busti@univr.it

10 Service d'Hématologie, Hôpital Pitié Salpêtrière, 47-83 boulevard de l'hôpital, F-75013 Paris, France; laurent.garderet@aphp.fr

11 Institute of Pathology, Catholic University of Sacred Heart, Largo A. Gemelli 8, 00168 Rome, Italy; maurizio.martini@unicatt.it

12 The University of Queensland Diamantina Institute, Translational Research Institute, 37 Kent Street Woolloongabba QLD 4102, Australia; r.dolcetti@uq.edu.au

13 Department of Medical \& Surgical Sciences, Magna Graecia University of Catanzaro, I-88100 Catanzaro, Italy; saderosa@unicz.it

14 Department of Hematology, University of Palermo, Vespro 127, I-90127 Palermo, Italy; sergio.siragusa@unipa.it

15 Department of Cellular Pathology, University College London, 21 University Street, London WC1E 6DE, UK; t.marafioti@ucl.ac.uk

16 Professor of Pathology, Former Chairman of Department of Pathology, Centro di Riferimento Oncologico (CRO), National Cancer Institute, Via Franco Gallini 2, 33081 Aviano, Italy

* Correspondence: acarbone@cro.it

$+\quad$ This Editorial has been prepared by the Editor-in-Chief (Antonino Carbone) and the Managing Editor (Carey Yuan) on behalf of the Editorial Board of Bloods.

Received: 19 March 2020; Accepted: 19 March 2020; Published: 20 March 2020

check for updates

Communication in the field of biomedical research today involves professionals of numerous backgrounds, including researchers, doctors, public administrators, politicians, and health journalists. Usually, biomedical researchers draw information from their own research data, while all other professionals use information from the scientific literature, institutional press releases, or uncontrolled 
sources to guide their professional activities and to create public opinion. During this whole process, the view of expert researchers-who act as a filter between biomedical research data and their potential impact on civil society-is missing.

Bloods intends to serve as the voice of a multidisciplinary network of authoritative scientists skilled in the field of hematology. Each quarterly issue of the journal will include a joint editorial authored by the Editor-in-Chief or by the Deputy Editor along with Section Editors. In these editorials, we highlight, in brief reports (of about one hundred words), a number of recently published articles that describe the most recent advancements in hematology. The editorial will be open as a forum with and for readers.

Therefore, the journal will not only publish high-level scientific articles, but will also discuss their significance in the scientific scenario, which includes the transfer of information to the media. Furthermore, for this reason, it is necessary that the sections into which the journal is divided are assigned to the care of a Section Editor. The Section Editor will comment on the application/operational aspect of the research published in the journal.

The Editor-in-Chief will be assisted by one or two Deputy Directors. There will be provisional sections (Non-Neoplastic Blood Disorders; Myeloid Leukemias; Lymphoid Leukemias; Lymphomas; and Plasma Cell Disorders Section), and each will be edited by a Section Editor. 